\title{
UCRL-JRNL-202692
}

\section{Complex Flows by Nanohydrodynamics}

\author{
W. E. Alley, P. Covello, and B. J. Alder
}

March 4, 2004

to be published in Molecular Physics 
This document was prepared as an account of work sponsored by an agency of the United States Government. Neither the United States Government nor the University of California nor any of their employees, makes any warranty, express or implied, or assumes any legal liability or responsibility for the accuracy, completeness, or usefulness of any information, apparatus, product, or process disclosed, or represents that its use would not infringe privately owned rights. Reference herein to any specific commercial product, process, or service by trade name, trademark, manufacturer, or otherwise, does not necessarily constitute or imply its endorsement, recommendation, or favoring by the United States Government or the University of California. The views and opinions of authors expressed herein do not necessarily state or reflect those of the United States Government or the University of California, and shall not be used for advertising or product endorsement purposes.

This work was performed under the auspices of the U.S. Department of Energy by University of California, Lawrence Livermore National Laboratory under Contract W-7405-Eng-48. 


\title{
Complex Flows by Nanohydrodynamics
}

\author{
W. Edward Alley, Paul Covello, and Berni Alder \\ Lawrence Livermore National Laboratory \\ P.O. box 808, Livermore, Cal. 94550
}

\begin{abstract}
The study of complex flows by particle simulations is speeded up over molecular dynamics (MD) by more than two orders of magnitude by employing a stochastic collision dynamics method (DSMC) extended to high density (CBA). As a consequence, a picture generated on a single processor shows the typical features of the Rayleigh-Taylor instability and is in quantitative agreement with the experimentally found long time behavior.
\end{abstract}

When researchers generally use the prefix "nano" they mean that they either have to study systems that are naturally small, as in biology, or they create small clusters of particles in the hope that they have desirable properties that differ from the bulk. Here the prefix nano is used differently, namely we are forced to study small systems in the hope that they behave as in bulk. The reason that we are forced to study small systems is that we want to simulate complex hydrodynamic phenomena by a massive particle dynamics calculation. Since even the biggest present computers can handle only about ten to the nine particles, ${ }^{(1)}$ this means that in three dimensions the system contains about 1000 particles on each side or is less than 1000 nanometers wide. Furthermore, we can pursue the dynamics for only a few nanoseconds in a reasonable amount of computer time, so that the prefix is doubly justified.

Why would one want to pursue such an endeavor? There are many good reasons and many indications that it might succeed. If successful, it would open up a whole new area for molecular dynamics exploration, such as, for example, atomistic mechanisms for the onset of turbulence. Prof. Powels, who, among many other things, has pioneered many novel applications of molecular dynamics to gain insight into physical processes, would, we are sure, greatly appreciate such an effort. To persuade him that this is not a foolish effort, it can be pointed out that a few hundred particles have accurately yielded equilibrium properties of macroscopic systems. ${ }^{(2)}$ Furthermore, the decay of fluctuations could be accurately described by continuum hydrodynamics at a distance scale of a few nanometers and ten to the minus three nanoseconds. ${ }^{(3)}$ What has not been tried up to now is whether one can also simulate chaotic behavior for such small systems in the time available.

The advantage of using particle methods rather than the usual continuum NavierStokes hydrodynamics approach are many. Let us use as an illustration the RayleighTaylor instability, which is the first instability we investigated. ${ }^{(4)}$ The instability is caused by a heavy fluid placed on top of a light fluid in a gravitational field, so that the heavy fluid penetrates the light fluid by spikes and in turn the light fluid forms bubbles in the heavy fluid. The first advantage of the particle approach is that the continuum approach can not deal accurately with the enormous concentration gradients that are initially present at the interface and subsequently persist at the edges of the bubbles 
and the spikes, nor can it deal with the huge shear gradients present at the boundaries. This is because Navier-Stokes contains only the linear terms in the applicable gradients. The expansion to higher order in the gradients diverges and so it has not been possible in practice to go beyond first order. Fortunately for Navier-Stokes hydrodynamics, it has been shown by non-equilibrium molecular dynamics ${ }^{(5)}$ that the imposition of enormous gradients does not have great quantitative consequences, although in some cases it can change the physics of the problem. A case in point is shear thinning if the applied stresses are too severe.

Another advantage of particle dynamics is that the natural fluctuations are present, which are averaged out in Navier-Stokes hydrodynamics. The natural fluctuations allow us to set up a natural rough interface initially, while in continuum calculations an artificial perturbation is imposed, and in experiments it is difficult to control the initial state. Furthermore, it seems that in Navier-Stokes calculations the coefficient describing long time behavior appears to depend on the amplitude and wavelength of this initially imposed perturbation, ${ }^{(6)}$ while such a perturbation superimposed on the fluctuations is washed out in particle simulations, provided the amplitude is not too large. For large amplitudes the particle simulations have not been carried out for long enough times ${ }^{(4)}$ to make the same claim, though we expect it to be true, because the fluctuations present eventually destroy the coherence of the initially imposed perturbation. The presence of fluctuations appears also to change the qualitative behavior at long times, equivalent to what has been shown to occur for a droplet forming from a dripping faucet. ${ }^{(7)}$ These droplets are connected to the flow before breaking off by a thin thread, that does not break in Navier-Stokes hydrodynamics, but does in molecular dynamics simulations because of the presence of fluctuations. A noise term added to the Navier-Stokes equation has been shown to lead to the breaking off as well. In Rayleigh-Taylor flow the situation is pretty similar, since at the tip of the spikes mushrooms form due to the KelvinHelmholtz instability on the sides. As the spike progresses, the tip of the spikes get thinner and the mushrooms are increasingly more tenuously connected to the tip. In one long calculation so far, the mushrooms were found to break off, forming droplets, resulting in a quantitative change in the character of the asymptotic behavior.

There are also great numerical advantages to using particle methods as opposed to Navier-Stokes solvers. The particle methods are gridless and unconditionally numerically stable. However, they are time consuming. This is not as bad as it first might appear, since the grid points required in a Navier-Stokes calculation ${ }^{(8)}$ is also about ten to the nine in order to get good spatial resolution. To advance a grid point is not all that much faster than advancing a particle position, so that the total calculating times are comparable. We have, however, found a much faster particle method that leads to much less time consuming calculations. This is the major new development first reported here.

Instead of using deterministic molecular dynamics, we employ a stochastic method used to solve exactly the full nonlinear Boltzmann equation. The algorithm called $\mathrm{DSMC}^{(9)}$ has long been used in aerodynamics. The algorithm takes advantage of the molecular chaos approximation and executes collisions of pairs of particles according to a known probability distribution. The problem for the Rayleigh-Taylor study is that this algorithm applies only at low densities where the instability occurs only at long 
times, if at all, because the two different perfect gases primarily just interdiffuse. Thus we need to extend this algorithm to higher densities, that we did some years ago in order to make the Boltzmann equation self consistent, called CBA; ${ }^{(10)}$ self consistent in that it gives the transport properties and the equation of state correct to first order in the density, while before the equation of state was always that of a perfect gas. In fact, this simple extension can be made to generate an arbitrary equation of state at any density, however, the calculated transport coefficients are not exact as the correlation between successive particle moves is neglected. For fluid flows, however, this does not matter as long as we know the transport coefficients and the molecular correlations ignored are of a much smaller time duration than the flow phenomena. In fact, the transport coefficients are quite realistic, and the model is comparable to one made a long time ago by Enskog ${ }^{(11)}$ to extend the Boltzmann equation to higher densities.

The preliminary attempts to use CBA for the Rayleigh-Talor instability have been quite successful. We have used a hard sphere system of two different masses because it runs even for molecular dynamics faster than the usual Lennard-Jones potential system by a factor of about 2.5. Table 1 lists how much faster the CBA system runs than molecular dynamics for 20000 hard sphere particles on the same single processor. Both algorithms have only been roughly optimized. The speed of the CBA algorithm is independent of density, here given in terms of the volume relative to the closed packed volume, V0, while the MD algorithm gets less efficient at lower densities, because the particles in the near neighborhood change more often. The primary reason for the speed up is avoiding the smallest time search for the next collision in CBA.

Table 1. Speed up of CBA over MD for hard spheres at various densities.

\begin{tabular}{|r|r|}
\hline V/V0 & Speed Up \\
\hline 1.6 & 41 \\
3.0 & 87 \\
5.0 & 136 \\
10.0 & 230 \\
20.0 & 396 \\
\hline
\end{tabular}

With this CBA algorithm we have been able to generate the instability as shown in Fig. 1 on a single workstation running at $900 \mathrm{MHz}$. for 60 hours with one million hard spheres, where the initial density at the top was $\mathrm{V} / \mathrm{V} 0$ of 3 with a mass ratio of 10 and a gravity of one in dimensionless units. The mushrooms on the spikes and the separation of a bubble can be clearly seen. In the previous MD study using the Lennard-Jones potential a similar computer experiment took similar time on a supercomputer with several hundred processors, because the program was several hundred times slower. A different run with 1.5 million hard spheres on a single $3 \mathrm{GHz}$. processor for an Atwood number $A$ (the ratio of the differences in the masses to the sum of the masses) of 0.8 and a V/V0 $=10$ at the top gave an asymptotic slope $\alpha$ for $g=1$ in $\alpha A g t^{2}$ within the error bars 
previously found. ${ }^{(4)}$

The CBA code is ideally suited for massively parallel computers and with this enhanced speed, we can confirm some of the suggestions, like the effects of fluctuations, made at the beginning of this paper. We can pursue the system for longer times and hopefully establish quantitatively the correct asymptotic behavior. We can, to some small extent, investigate the effect of a reduced huge gravitational field needed to scale the instability to the nanoscale, but the value of $g$ will always be many order of magnitudes higher than in the real world, and so dependence on the validity of scaling will always remain an open question, but we are in good company, since Navier-Stokes assumes it as well. In sum, we are embarking on an alternate way to compute complex flows, and hopefully it helps resolve some of the long outstanding questions in this classical field that have resisted probing.

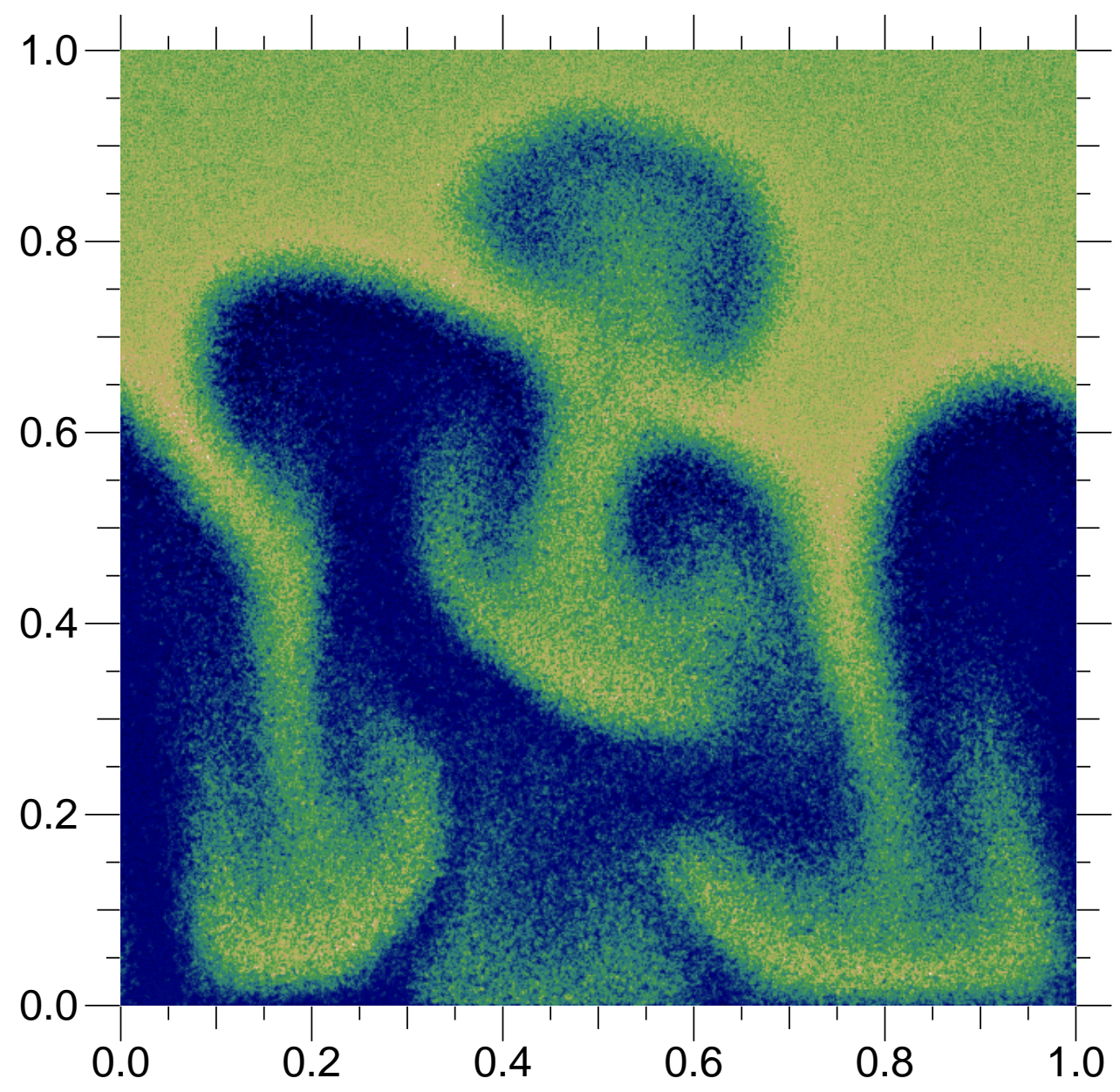

Fig.1 Demonstration of the Rayleigh-Taylor instability. The color variation goes from yellow at the top for the highest density to dark blue for the lowest density. 


\section{REFERENCES}

1. K. Kadau, T. C. Germann and P. S. Lomdahl, Int. J. Mod. Phys. C15, in press (2004)

2. B. J. Alder and T. E. Wainwright, J. Chem. Phys. 27, 1208 (1957)

3. B. J. Alder and T. E. Wainwright, Phys. Rev. A1, 18 (1970)

4. K. Kadau, T. C. Germann, N. C. Hadjiconstantinou, P. S. Lomdahl, G. Dimonte, B. L. Holian and B. J. Alder, in press (2004)

5. W. G. Hoover and W. T. Ashurst, Adv. Theor. Chem. 1, 1 (1975)

6. G. Dimonte, Phys. Rev. E, in press (2004)

7. M. Moseler and U. Landman, Science 289, 1165 (2000)

8. A. W. Cook, W. Cabot and P. L. Miller, J. Fluid Mech., in press (2004)

9. G. Bird, Molecular Gas Dynamics and the Direct Simulation of Gas Flow, Clarendon, Oxford (1994)

10. F. Alexander, A. Garcia and B. Alder, Phys. Rev. Letters 74, 5212 (1995)

11. S. Chapman and T. G. Cowling, The Mathematical Theory of Nonuniform Gases, Cambridge University Press (1939) 